\title{
Análisis de Algunos Aspectos del Juicio Oral Mercantil a Tres Años de su Entrada en vigor en Nuestra Entidad.
}

\section{Analysis of some aspects of the trial Oral trading company to three years of its entry into force in our State.}

\author{
Héctor Manuel Murguía Murguía ${ }^{1}$
}

SUMARIO: I.- Introducción. II.- La acción como derecho subjetivo público. III.Sentencias definitivas, IV.- Síntesis o conclusiones.

Juicio oral mercantil

\section{Resumen}

Breve análisis de ciertos aspectos de la oralidad en materia mercantil derivada de la reforma al Código de Comercio al que se le adiciona el TíTULO ESPECIAL denominado "DEL JUICIO ORAL MERCANTIL"(Artículos 1390 BIS y del 1390 BIS-1 al 1390 BIS-50) preceptos que estructuran esta modalidad en la resolución de ciertas controversias mercantiles, el cual entró en vigor al año siguiente de su publicación, en el DOF (Diario Oficial de la Federación) el día 27 de enero de 2011.

Palabras clave : juicio oral mercantil,controversias mercantiles

\section{Abstract}

Brief analysis of certain aspects of the orality in commercial matter derived from the Commercial Code Reform to which was added the title Special called "of the commercial oral trial" (Articles 1390 BIS and $1390 \mathrm{BIS}-1$ to the $1390 \mathrm{BIS}-50$ ) precepts that regulate this mode in the resolution of certain commercial disputes, which entered in force to the following year of its publication, in the DOF (Official Journal of the Federation ) of January 27, 2011.

Key words: commercial oral trial, resolution of commercial disputes

\section{Introducción}

El análisis que nos ocupa, hace obligada referencia a los conceptos de jurisdicción y competencia y al respecto, Francisco José Contreras Vaca establece que la primera "es la facultad soberana del Estado para conocer y resolver con fuerza vinculativa para las partes una controversia sometida a proceso o, en su caso, reconocer la validez y

\footnotetext{
${ }^{1}$ Docente de la Universidad de Sonora, integrante de la Academia de Derecho Mercantil, Candidato a Doctor por el Centro de Estudios Superiores en Ciencias Jurídicas y Criminológicas Unidad de Enlace Sonora.
} 
ejecutar decisiones emitidas por otra autoridad jurisdiccional, siempre que se satisfagan los requisitos exigidos por su legislación" y la segunda, la competencia, determina que "es el límite de la jurisdicción" y así tenemos que existe, por ejemplo, competencia de grado, materia y territorio.

En esta tesitura, la competencia para conocer y resolver válidamente conflictos orales mercantiles, la determinan:

A).- La cuantía, que el monto de lo reclamado por concepto de suerte principal sea inferior a la cantidad de $\$ \mathbf{5 6 2 , 2 6 4 . 4 3}$ (SON: QUINIENTOS SESENTA Y DOS MIL DOSCIENTOS SESENTA Y CUATRO 43/100 M.N.) que determina el artículo 1309 BIS y que remite al diverso numeral 1339, ambos del Código de Comercio,

B).- Que no haya indicación expresa de tramitación especial, como sería el caso del procedimiento judicial de ejecución de garantías otorgadas mediante prenda sin transmisión de posesión y fideicomiso de garantía, reglamentado en el CAPÍTULO II, artículos del 1414 BIS-7 al 1414 BIS-20, del Código de Comercio y,

C).- Que no sean de cuantía indeterminada, según lo previene el artículo 1390 BIS-1 del Código de Comercio.

Ahora bien, la oralidad no es un sistema completamente nuevo, ya que nuestro Código de Procedimientos Civiles para el Estado de Sonora, que entró en vigor en el mes de septiembre de 1949, en su Capítulo lii reglamenta el juicio oral, artículos del 500 al 507, determinando, que tanto la demanda, la contestación y la sentencia son escritas, dándose si la oralidad, en la recepción y práctica de las pruebas e incidentes que surjan en el juicio. 
La referencia contenida en el párrafo que antecede, resulta de vital importancia toda vez que esencialmente sucede lo mismo en el juicio oral mercantil que nos ocupa, es decir, la demandada, contestación, reconvención, contestación a la misma, el acta que se levanta al terminar las audiencias y también el acto procesal de mayor relevancia, la sentencia definitiva, son escritos, según lo establecen los artículos 1390 BIS-11, 1390 BIS-14, 1390 BIS-16, 1390 BIS-17, 139 BIS-18, 1390 BIS-27, 1390 BIS-38 párrafo segundo, 1390 BIS-39 del Código de Comercio, observándose en forma especial los principios de oralidad, publicidad, igualdad, inmediación, contradicción, continuidad y concentración; estos tres últimos con el propósito de dar celeridad al proceso para resolver de fondo la controversia mediante la sentencia definitiva vinculatoria para las partes, con la salvedad de que no existen recursos ordinarios, lo que implica que tal resolución será impugnada mediante el amparo directo.

Efectivamente, si bien es cierto que en el juicio oral mercantil que nos ocupa no existen recursos ordinarios para impugnar las resoluciones judiciales, también es muy cierto que el Juez tiene amplísimas facultades para encausar apropiadamente el procedimiento, contenidas en el artículo 1390 BIS párrafos tercero y cuarto del Código de Comercio, por lo que podríamos pensar que se trata del recurso de revocación encubierto, que permite al Juez de oralidad subsanar ciertos errores procesales y además, cuenta con otro instrumento procesal de aclaración o adición a las resoluciones.

\section{La Acción como Derecho Subjetivo Público}

La palabra acción tiene en el derecho procesal múltiples significados y al respecto, el autor José Ovalle Favela nos indica que para Couture, ésta tiene cuando menos tres acepciones:

1.- "En primer lugar, se le utiliza como sinónimo del derecho subjetivo material que trata de hacerse valer en juicio...". 
2.- "La palabra acción también puede ser usada para designar la pretensión que la parte actora o acusadora formula en su demanda o en su acusación...".

3.- "Por último, la acción también es entendida como la facultad (o el derecho público subjetivo) que las personas tienen para promover un proceso ante un órgano jurisdiccional, con el fin de que, al concluir el proceso, emita una sentencia sobre una pretensión litigiosa y, en su caso, ordene la ejecución de la sentencia..." .

Ahora bien, al existir un derecho subjetivo, también debe existir la obligación a cargo de una persona, bien sea física o moral, entendida, la obligación, según el autor Rafael Rojina Villegas como "un vínculo jurídico por virtud del cual una persona denominada deudor, se encuentra constreñida jurídicamente a ejecutar algo en favor de otra persona, llamada acreedor".

Al respecto el autor Manuel Bejarano Sánchez define a la obligación, como: "la necesidad jurídica que tiene la persona llamada deudor, de conceder a otra llamada acreedor una prestación de dar, de hacer o de no hacer".

Tema toral del derecho civil es el relacionado con las fuentes generales de las obligaciones y al respecto, el Código Civil Federal nos indica que son los hechos y los actos jurídicos que reglamenta en los artículos del 1792 al 1937, numerales estos que por disposición expresa, entre otros, del artículo 1056 del Código de Comercio, resultan aplicables supletoriamente a la materia mercantil, pero que nuestro Código Civil Sonorense hace de las mismas una mejor reglamentación y clasificación en sus artículos del 1906 al 1922. 
Ahora bien, al tenor de las disposiciones antes referidas, el incumplimiento de una obligación, ya sea de hacer, no hacer o dar, está clasificado como un hecho jurídico voluntario ilícito que actualiza la hipótesis del reclamo del derecho subjetivo preterido, es decir, el cobro, con la salvedad de que en nuestro orden constitucional mexicano queda estrictamente prohibido hacerse justicia por propia mano, prohibición claramente contenida en el artículo 17 de nuestra Constitución General de la República Mexicana, al igual que en los códigos procesales del país, por lo que en la hipótesis de la falta de pago o incumplimiento de una obligación mercantil derivada de los actos de comercio que en forma enunciativa están contenidos en el artículo 75 del Código de Comercio y otras leyes mercantiles, el sujeto activo, titular del derecho de cobro, bajo los supuestos de competencia debe ocurrir ante el Tribunal de Oralidad a ejercer la acción correspondiente, la cual toma su nombre del contrato o hecho del que deriva, según el artículo 14 del Código de Procedimientos Civiles del Estado de Sonora de aplicación supletoria a la materia mercantil por disposición expresa del artículo 1054 del Código de Comercio y que va encaminada, por ejemplo, a que se condene al demandado a realizar una determinada prestación (dar, hacer y no hacer) a que se refiere la fracción I del artículo 13 del Código Adjetivo Sonorense.

La acción mercantil oral debe formularse por escrito mediante la demanda, que es considerada como un acto jurídico que normalmente da inicio al proceso para obtener del juzgador una resolución definitiva a la controversia que se le plantea y como tal, debe cumplir con la formalidad procesal, es decir, satisfacer los requisitos contenidos en el artículo 1390 BIS-11 del Código de Comercio y en ninguna de sus nueve fracciones mandata que se exprese la causa petendi, es decir, la causa de pedir, que no es otra cosa que indicar claramente la causa o causas generadoras del derecho subjetivo de exigirle al sujeto pasivo el pago o cumplimiento de su obligación.

Al parecer el artículo 1390 BIS-11 que se comenta, contiene una laguna de naturaleza adjetiva o procesal que origina o puede originar un estado de indefensión en el demandado para preparar adecuadamente su contestación de demanda y sobre todo para estructurar adecuadamente sus excepciones o defensas, por lo que en esta 
tesitura debe aplicarse en primer término, en forma supletoria, el Código Federal de Procedimientos Civiles, que en su artículo 322 fracción III, establece que el actor debe narrar los hechos en que funde su petición (causa petendi) la cual más o menos concuerda con la fracción VI del artículo 227 del Código de Procedimientos Civiles para el Estado de Sonora, pero se considera que este último precepto es más claro y preciso al exigir que en la demanda quede establecido cuál es el título o la causa de la acción que se ejercita, ordenamientos jurídicos adjetivos que resultan aplicables a la materia mercantil al tenor, entre otros, del artículo 1054 del Código de Comercio.

Es muy probable que surja la confusión entre la causa petendi ${ }^{2}$ contenida en aquella parte de la fracción VI del artículo 227 que literalmente establece: “..., y quede establecido cuál es el título o la causa de la acción que se ejercite," y la acción causal que es de naturaleza mercantil contemplada en el artículo 168 de la Ley General de Título y Operaciones de Crédito, que contempla la hipótesis de que la acción cambiaria haya prescrito o caducado, por lo que la causa petendi viene a constituir el género y la acción causal, la especie.

Efectivamente la causa petendi es un concepto genérico relativo a las fuentes generales de las obligaciones, que como ya se ha manifestado puede provenir de un hecho o bien de un acto jurídico y por el contrario, la acción causal a que alude el precitado artículo 168 de la Ley General de Título y Operaciones de Crédito, siempre e invariablemente se refiere a un acto jurídico de naturaleza mercantil, de los enumerados en las diversas fracciones del artículo 75 del Código de Comercio y en otras leyes mercantiles como causa de la emisión o transmisión de la letra de cambio o bien del pagaré, no siendo esta acción de naturaleza ejecutiva y por lo tanto, de

\footnotetext{
${ }^{2}$ Causa de pedir, es decir, los motivos que originan el ejercicio de una acción, los cuales pueden calificarse en supuestos de hecho previstos en el ordenamiento jurídico, cuyas consecuencias jurídicas fundamentan el petitum del proceso. Con esta expresión, que literalmente significa causa de pedir, se alude al título o fundamento de la pretensión procesal que se formula en la demanda y que constituye uno de los elementos básicos de la reclamación judicial que formula el demandante. Este debe describir al tribunal el conjunto de hechos que, enlazados entre sí, forman la unidad fáctica sobre la que se apoya la reclamación del actor. Al acudir al órgano jurisdiccional, el reclamante tiene que proporcionar hechos para que el tribunal le reconozca derechos; como se ha dicho desde antiguo, da mihi factum, dabo tibi ius (dame hecho, y te daré derecho), como expresión de la esencia de la actividad jurisdiccional. La causa de pedir o reclamar a un tribunal está en una zona de la actividad humana de la que, hasta el momento de formularse la pretensión, no tenía noticia el tribunal. Enciclpedia Jurídica [online] Disponible en: http://www.enciclopedia-juridica.biz14.com/d/causa-petendi/causa-petendi.htm
} 
acuerdo a las hipótesis que se actualicen será competencia, bien, del juicio ordinario u oral mercantiles que por no existir indicación expresa de prescripción menor, debe aplicarse la prescripción ordinaria de 10 años contemplada en el artículo 1047 del Código de Comercio.

En este contexto, resulta que un gran porcentaje de abogados postulantes al ocurrir al Juzgado Oral Mercantil con residencia en esta ciudad de Hermosillo, Sonora, se desatienden de la formalidad que debe contener la demanda, omitiendo tanto la denominación de la acción, como el título o la causa de la referida acción que se ejercita. Por su parte, el órgano jurisdiccional no debería radicarla y en todo caso, pedirle al actor la aclare en el término de tres días según lo establece el artículo 1390 BIS-12 del Código de Comercio.

Asimismo, las acciones más comúnmente ejercitadas ante el Juez de Oralidad están sustentadas en títulos de crédito, concretamente en el pagaré, que si bien contiene cantidad determinada, líquida y exigible, la acción cambiaria directa a que se refiere el artículo 167 de la Ley General de Títulos y Operaciones de Crédito prescribió por no haberla ejercitado en el término o plazo de tres años (regla general) que establece el diverso numeral 165 de esta codificación por lo que en esta hipótesis, este documento deja de ser ejecutivo tornándose en un documento meramente demostrativo o confesorio, por lo que su tenedor beneficiario válidamente puede demandar mediante la acción causal contenida en el artículo 168:

A).- El cumplimiento o recisión del acto jurídico, contrato que fue la causa por la cual se firmó el título de crédito.

Por lo que toca a la recisión, seria competencia del ordinario mercantil por ser esta acción de cuantía indeterminada. Si se demanda el cumplimiento forzoso del contrato, dependiendo de la cuantía que por concepto de suerte principal se reclame, sería el ordinario o bien el oral, y 
B).- La acción de pago de dinero, es decir, de pesos mexicanos, dólares norteamericanos, euros, etc. y que se desprende del propio título de crédito prescrito que contiene literalmente una promesa de pagar una suma determinada de dinero en un lugar y tiempo determinados, lo que implica que el juicio oral que nos ocupa, no queda limitado jurisdiccionalmente únicamente para conocer de la acción causal sino también para conocer de la acción de pago de dinero.

Para consolidar las argumentaciones y conclusiones que anteceden, se pueden actualizar hipótesis en que el Juez de Oralidad sea competente para conocer y resolver controversias mercantiles en donde: A) La acción causal no exista, o B) Que existiendo quede prohibido su ejercicio contra el suscriptor original.

La primera de las hipótesis enunciadas, que la acción causal no exista, está contemplada en el propio artículo 168 de la Ley General de Títulos y Operaciones de Crédito, al establecer que esta acción no existe en el caso de que haya Novación.

Ley, doctrina y jurisprudencia son uniformes al considerar que la causa de suscripción o transmisión de un título de crédito, el más utilizado y consecuentemente conocido, el pagaré, es que subyace el acto jurídico por excelencia, el contrato y así tenemos que efectivamente, al celebrar un acto jurídico de naturaleza mercantil, por ejemplo, el de Apertura de Crédito que es de naturaleza bilateral generador de derechos y obligaciones recíprocos, facultando al acreditante a solicitarle al acreditado la firma o suscripción de un pagaré por el monto del crédito dispuesto por lo que al firmarlo, se crean dos relaciones jurídicas, una de naturaleza contractual y la otra de naturaleza cambiaria, lo que implica una duplicidad de obligaciones y ante tal evento, el acreditado al aceptar suscribir el título de crédito, acuerda o conviene con su acreedor, el acreditante, que las obligaciones derivadas del contrato de Apertura de Crédito, se extingan mediante la Novación y en esta hipótesis y ante el incumplimiento de pago del título de crédito y prescrita su acción cambiaria, el tenedor beneficiario del mismo, precisamente por carecer de acción cambiaria y de acción causal por haberse 
extinguido esta última por novación, lo conducente a ejercitar será la acción de pago de dinero, ya sea pesos, dólares norteamericanos, etc., ante el Juez de Oralidad, satisfechas las hipótesis de competencia.

La segunda de las hipótesis, relativa a que bajo ciertos supuestos, aun existiendo la acción causal a que se refiere el artículo 168 quede prohibido su ejercicio contra el suscriptor original; para explicar la hipótesis que nos ocupa, volvemos al ejemplo del título de crédito más conocido y usado que es el pagaré, que al ser endosado en propiedad y prescrita la acción cambiaría directa por no haberla ejercido dentro de los tres años siguientes al vencimiento (regla general), su tenedor beneficiario no podrá invocar la causa por la cual se le suscribió el referido pagaré a su endosante, pero si estará legitimado para hacer valer contra el suscriptor original del pagaré prescrito, la acción de pago de dinero, ya sea pesos, dólares norteamericanos, etc. por qué en última instancia, la literalidad contenida en el referido título de crédito prescrito es la promesa incondicional de pagar una suma determinada de dinero.

Sirve de sustento a esta segunda hipótesis la siguiente jurisprudencia que se transcribe:

Época: Décima Época

Registro: 2005033

Instancia: Primera Sala

Tipo de Tesis: Jurisprudencia

Fuente: Gaceta del Semanario Judicial de la Federación

Libro 1, Diciembre de 2013, Tomo I

Materia(s): Civil

Tesis: 1a./J. 96/2013 (10a.)

Página: 291

ACCIÓN CAUSAL. EL ENDOSATARIO EN PROPIEDAD DE UN TÍTULO DE CRÉDITO NO PUEDE EJERCERLA CONTRA EL SUSCRIPTOR ORIGINAL. Del artículo 168 de la Ley General de Títulos y Operaciones de Crédito se advierte que la acción causal es independiente de la acción cambiaria, al subsistir si ésta se pierde, siempre que derive del negocio que originó el título de crédito o su transmisión y que no hubiera novación. Lo 
anterior, porque la emisión o transmisión de los títulos de crédito proviene de un negocio jurídico subyacente, lo que implica que por cada endoso se generan diversas relaciones jurídicas entre endosatarios y endosantes. Por tanto, para que el suscriptor de un título de crédito pueda ser demandado mediante una acción causal, es requisito que haya sido parte en el negocio jurídico del cual deriva dicha acción, con base en el principio res inter alios acta (relatividad de los contratos). En consecuencia, el endosatario en propiedad de un título de crédito no puede ejercer acción causal contra el suscriptor original, toda vez que éste carece de legitimación pasiva para ser demandado por el último tenedor, ya que no comparten una relación jurídica causal entre ellos, lo cual además contrasta con las acciones cambiarias, que emanan de los títulos de crédito que pueden exigirse contra el deudor original en la vía directa o contra los demás signatarios en vía de regreso.

Contradicción de tesis 170/2013. Entre las sustentadas por el Tercer Tribunal Colegiado en Materia Civil del Primer Circuito y el Primer Tribunal Colegiado del Décimo Octavo Circuito. 28 de agosto de 2013. La votación se dividió en dos partes: mayoría de cuatro votos por la competencia. Disidente: José Ramón Cossío Díaz. Unanimidad de cinco votos en cuanto al fondo. Ponente: Olga Sánchez Cordero de García Villegas. Secretaria: Rocío Balderas Fernández.

Tesis de jurisprudencia 96/2013 (10a.). Aprobada por la Primera Sala de este Alto Tribunal, en sesión de fecha once de septiembre de dos mil trece.

Esta tesis se publicó el viernes 6 de diciembre de 2013 a las 06:00 horas en el Semanario Judicial de la Federación y, por ende, se considera de aplicación obligatoria a partir del lunes 9 de diciembre de 2013, para los efectos previstos en el punto séptimo del Acuerdo General Plenario 19/2013.

\section{Sentencias Definitivas}

El proceso, definido por José Ovalle Favela como "el conjunto de actos mediante los cuales se constituye, desarrolla y termina la relación jurídica que se establece entre el juzgador, las partes y las demás personas que en ella intervienen; y que tienen como finalidad dar solución al litigio planteado por las partes, a través de una decisión del juzgador basada en los hechos afirmados y probados y en el derecho aplicable". 
Al concluir esa secuencia formal de actos que conforman al proceso, se llega a la etapa resolutiva que viene a constituir el acto jurídico procesal de máxima relevancia, que es la sentencia definitiva. Definida por Francisco José Contreras Vaca como "el acto procesal en virtud del cual el tribunal resuelve los puntos litigiosos sometidos a debate en uso de la facultad jurisdiccional delegada por el Estado".

Estas resoluciones de fondo deben ser siempre apegadas a derecho, razón demás, si como consecuencia de la reforma del 2008 al artículo $1^{\circ}$ Constitucional se introduce el concepto de derechos humanos que otorga nuestra Constitución y los Tratados Internacionales, entre otros, la Convención Americana de Derechos Humanos, "Pacto de San José de Costa Rica" de la cual México es signatario y por lo tanto, vinculado a las resoluciones que emita el Tribunal Interamericano de Derecho Humanos y entre ellas, el derecho humano al debido proceso, por lo que aún en el supuesto de que la norma nacional no otorgue las debidas garantías para la preservación de estos derechos, todo juez nacional o doméstico deberá dejar de aplicarlos y en su lugar, aplicar la norma extranjera en base al principio pro persona por medio del control difuso de convencionalidad ex oficio y en esta tesitura, no obstante que las codificaciones adjetivas nacionales establezcan que la acción proceda en juicio aunque no se denomine o denomine incorrectamente, el órgano jurisdiccional deberá ser cuidadoso en aras de respetar el debido proceso, de que se denominen apropiadamente en los escritos de demanda y sobre todo, que en la misma, se precise el título o la causa de la acción que se ejercita para evitar vulnerar derechos humanos.

\section{Síntesis o Conclusiones}

Las universidades que ofertan la licenciatura en derecho, deberán ser muy cuidadosas de incluir en su plan de estudios aquellas materias jurídico formativas tanto de derecho sustantivo como adjetivo, para que egresen profesionistas con una formación jurídica sólida, con principios éticos, con aptitud plena para conocer e interpretar adecuadamente los problemas jurídicos que se le planteen y logrado esto, estructurar una demanda o bien, su contestación, en las cuales se cumplan con las formalidades que determina la ley y las aportaciones de las pruebas conducentes, para que el Juez pueda emitir una sentencia definitiva apegada a derecho y con esto se creara una 
situación de seguridad que atraerá inversionistas que generen fuentes de trabajo en beneficio de la sociedad sonorense y en cabal respeto a los derechos humanos.

\section{Bibliografía}

- BEJARANO SÁNCHEZ,Manuel, “Obligaciones Civiles”, Sexta edición, Oxford,2007.

- CONTRERAS VACA, Francisco José ,Derecho Procesal Civil, edición, Oxford ,2007.

- OVALLE FAVELA,José, Teoría General del Proceso, Oxford, edición,Oxford, 2005.

- ROJINA VILLEGAS, Rafael,Compendio de Derecho Civil, Teoría General de las Obligaciones, Décima novena edición, Editorial Porrúa, S.A. 1994.

\section{Legislación}

- Código Civil Federal vigente a partir del el $1^{\circ}$ de octubre de 1932.

- Código Civil para el Estado de Sonora vigente a partir del 8 de julio de 1949.

- Código de Comercio vigente a partir de día $1^{\circ}$ de enero de 1890.

- Código de Procedimientos Civiles para el Estado de Sonora publicado el $1^{\circ}$ de agosto de 1949 en el Boletín Oficial.

- Ley General de Títulos y Operaciones de Crédito vigente a partir del 15 de septiembre de 1932

\section{Webgrafía}

- Enciclopedia Jurídica [online] Disponible en:

http://www.enciclopediajuridica.biz14.com/d/causa-petendi/causa-petendi.htm 